\title{
Femtosecond diffractive imaging with a soft-X-ray free-electron laser
}

HENRY N. CHAPMAN ${ }^{1,2 *}$, ANTON BARTY ${ }^{1}$, MICHAEL J. BOGAN ${ }^{1}$, SÉBASTIEN BOUTET $1,3,4$, MATTHIAS FRANK ${ }^{1}$, STEFAN P. HAU-RIEGE ${ }^{1}$, STEFANO MARCHESIN ${ }^{1,2}$, BRUCE W. WOODS ${ }^{1}$, SAŠA BAJT ${ }^{1}$, W. HENRY BENNER ${ }^{1}$, RICHARD A. LONDON ${ }^{1,2}$, ELKE PLÖNJES ${ }^{5}$, MARION KUHLMANN 5 , ROLF TREUSCH ${ }^{5}$, STEFAN DÜSTERER ${ }^{5}$, THOMAS TSCHENTSCHER ${ }^{5}$, JOCHEN R. SCHNEIDER ${ }^{5}$, EBERHARD SPILLER ${ }^{6}$, THOMAS MÖLLER ${ }^{7}$, CHRISTOPH BOSTEDT ${ }^{7}$, MATTHIAS HOENER ${ }^{7}$, DAVID A. SHAPIRO ${ }^{2}$, KEITH 0. HODGSON ${ }^{3}$, DAVID VAN DER SPOEL ${ }^{4}$, FLORIAN BURMEISTER ${ }^{4}$, MAGNUS BERGH ${ }^{4}$, CARL CALEMAN ${ }^{4}$, GÖSTA HULDT ${ }^{4}$, M. MARVIN SEIBERT ${ }^{4}$, FILIPE R. N. C. MAIA ${ }^{4}$, RICHARD W. LEE ${ }^{1,4}$, ABRAHAM SZÖKE ${ }^{1,4}$, NICUSOR TIMNEANU ${ }^{4}$ AND JANOS HAJDU $3,4 *$

\footnotetext{
${ }^{1}$ University of California, Lawrence Livermore National Laboratory, 7000 East Avenue, Livermore, California 94550, USA

${ }^{2}$ Center for Biophotonics Science and Technology, University of California, Davis, 2700 Stockton Blvd, Suite 1400, Sacramento, California 95817, USA

${ }^{3}$ Stanford Synchrotron Radiation Laboratory, Stanford Linear Accelerator Center, 2575 Sand Hill Road, Menlo Park, California 94305, USA

${ }^{4}$ Laboratory of Molecular Biophysics, Department of Cell and Molecular Biology, Uppsala University, Husargatan 3, Box 596, SE-75124 Uppsala, Sweden

${ }^{5}$ Deutsches Elektronen-Synchrotron, DESY, Notkestraße 85, D-22607 Hamburg, Germany

${ }^{6}$ Spiller X-ray Optics, Livermore, California 94550, USA

${ }^{7}$ Institut für Optik und Atomare Physik, Technische Universität Berlin, Hardenbergstraße 36, PN 3-1, 10623 Berlin, Germany

*e-mail: henry.chapman@IInl.gov; janos.hajdu@xray.bmc.uu.se
}

T heory predicts ${ }^{1-4}$ that, with an ultrashort and extremely bright coherent X-ray pulse, a single diffraction pattern may be recorded from a large macromolecule, a virus or a cell before the sample explodes and turns into a plasma. Here we report the first experimental demonstration of this principle using the FLASH soft-X-ray free-electron laser. An intense $25 \mathrm{fs}, 4 \times 10^{13} \mathrm{~W} \mathrm{~cm}^{-2}$ pulse, containing $10^{12}$ photons at $32 \mathrm{~nm}$ wavelength, produced a coherent diffraction pattern from a nanostructured non-periodic object, before destroying it at $60,000 \mathrm{~K}$. A novel X-ray camera assured single-photon detection sensitivity by filtering out parasitic scattering and plasma radiation. The reconstructed image, obtained directly from the coherent pattern by phase retrieval through oversampling ${ }^{5-9}$, shows no measurable damage, and is reconstructed at the diffraction-limited resolution. A three-dimensional data set may be assembled from such images when copies of a reproducible sample are exposed to the beam one by one ${ }^{10}$.

X-ray free-electron lasers (FELs) are expected to permit diffractive imaging at high resolutions of nanometre- to micrometre-sized objects without the need for crystalline periodicity in the sample ${ }^{1-4}$. Structural studies within this size domain are particularly important in materials science, biology and medicine. Radiation-induced damage and sample movement prevent the accumulation of high-resolution scattering signals for such samples in conventional experiments ${ }^{11,12}$. Damage is caused by energy deposited into the sample by the very probes used for imaging, for example photons, electrons or neutrons. At $\mathrm{X}$-ray frequencies, inner-shell processes dominate the ionization of the sample; photoemission is followed by Auger or fluorescence emission and shake excitations. The energies of the ejected photoelectrons, Auger electrons and shake electrons differ from each other, and these electrons are released at different times, but within about ten femtoseconds, following photoabsorption ${ }^{1,13}$. Thermalization of the ejected electrons through collisional electron cascades is completed within 10-100 fs (refs 14,15). Heat transport, diffusion and radical reactions take place over some picoseconds to milliseconds.

The effect of X-ray-induced sample damage on the recorded image or diffraction pattern could be substantially reduced, if we could collect diffraction data faster than the relevant damage processes $^{1,16}$. This approach requires very short and very bright $\mathrm{X}$-ray pulses, such as those expected from a short-wavelength FEL. However, the large amount of energy deposited into the sample by a focused FEL pulse will ultimately turn the sample into a plasma. The question is when exactly would this happen. There are no experiments with X-rays in the relevant time and intensity 


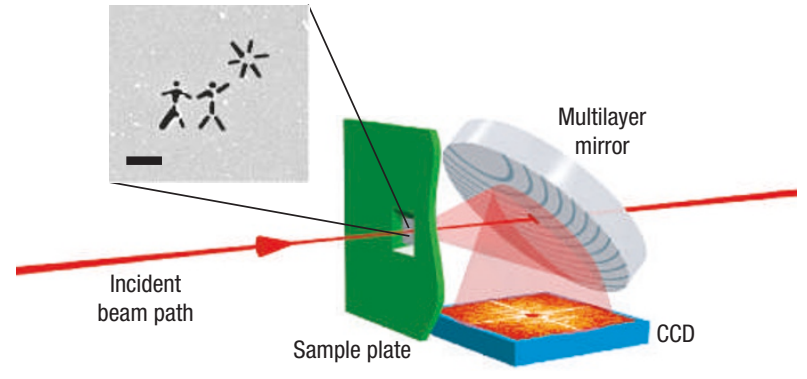

Figure 1 Schematic diagram of the experimental apparatus. The FEL beam is incident from the left and is focused to a $20 \mu \mathrm{m}$ spot on the sample, which is a 20-nm-thick transmissive silicon nitride membrane with a picture milled through its entire thickness using an FIB (this is enlarged in the inset, and the scale bar indicates $1 \mu \mathrm{m})$. The direct beam passes through the sample window and exits the camera through a hole in a graded multilayer planar mirror. The diffracted light from the sample reflects from this mirror onto a CCD detector. The contour lines on the mirror depict lines of constant incidence angle (constant multilayer period). The on-axis path length from the sample to the detector is $55 \mathrm{~mm}$. For $32 \mathrm{~nm}$ radiation and objects smaller than $20 \mu \mathrm{m}$, this distance is in the far field, where the diffraction pattern is equal to the Fourier transform of the exit wave ${ }^{27}$. The numerical aperture of the detector is 0.25 .

domains, and our current understanding of photon-material interactions on ultrashort timescales and at high X-ray intensities is, therefore, limited. Computer simulations on the basis of four different models ${ }^{1-4}$ postulate that a near-atomic-resolution structure could be obtained by judicious choice of pulse length, intensity and X-ray wavelength, before the sample is stripped of its electrons and is destroyed in a Coulomb explosion. Near-atomic-resolution imaging with X-ray FEL pulses faces other formidable challenges that must be addressed, such as developing the ability to record low-noise and interpretable diffraction data under the extreme illumination conditions expected from a focused FEL pulse.

Our experimental demonstration of 'flash diffractive imaging' uses the first soft-X-ray FEL in the world, the FLASH facility (formerly known as the VUV-FEL) at the Deutsches ElektronenSynchrotron in Hamburg ${ }^{17}$. FLASH generates high-power soft-Xray pulses by the principle of self-amplification of spontaneous emission $^{18}$ : a relativistic electron pulse from a superconducting linear accelerator makes a single pass through a periodic magnetic field of an undulator. During the high-gain lasing process, the electrons, perturbed by the magnetic field of the undulator and by their own photon field, form coherent microbunches, which behave like a single giant charge, producing strong amplification. For our experiment, FLASH was operating in an ultrashort-pulse mode $^{17}$, resulting in $25 \mathrm{fs}$ coherent FEL pulses, which were close to transform limited (as determined from spectral measurements of the FEL pulses and the measurement of shot-to-shot intensity fluctuations), and contained about $10^{12}$ photons in a pulse.

Figure 1 shows our experimental arrangement. Diffractive imaging is elegant in its simplicity: a coherent X-ray beam illuminates the sample, and the far-field diffraction pattern of the object is recorded on an area detector. We focused a coherent 25 fs X-ray pulse from FLASH to achieve a peak intensity of $(4 \pm 2) \times 10^{13} \mathrm{~W} \mathrm{~cm}^{-2}$ on the sample. We recorded the far-field diffraction pattern of the object on a novel detector centred on the forward direction (see the Methods section). The image information encoded in the coherent diffraction pattern is similar to a hologram ${ }^{19}$, except that the object acts as its own scattering
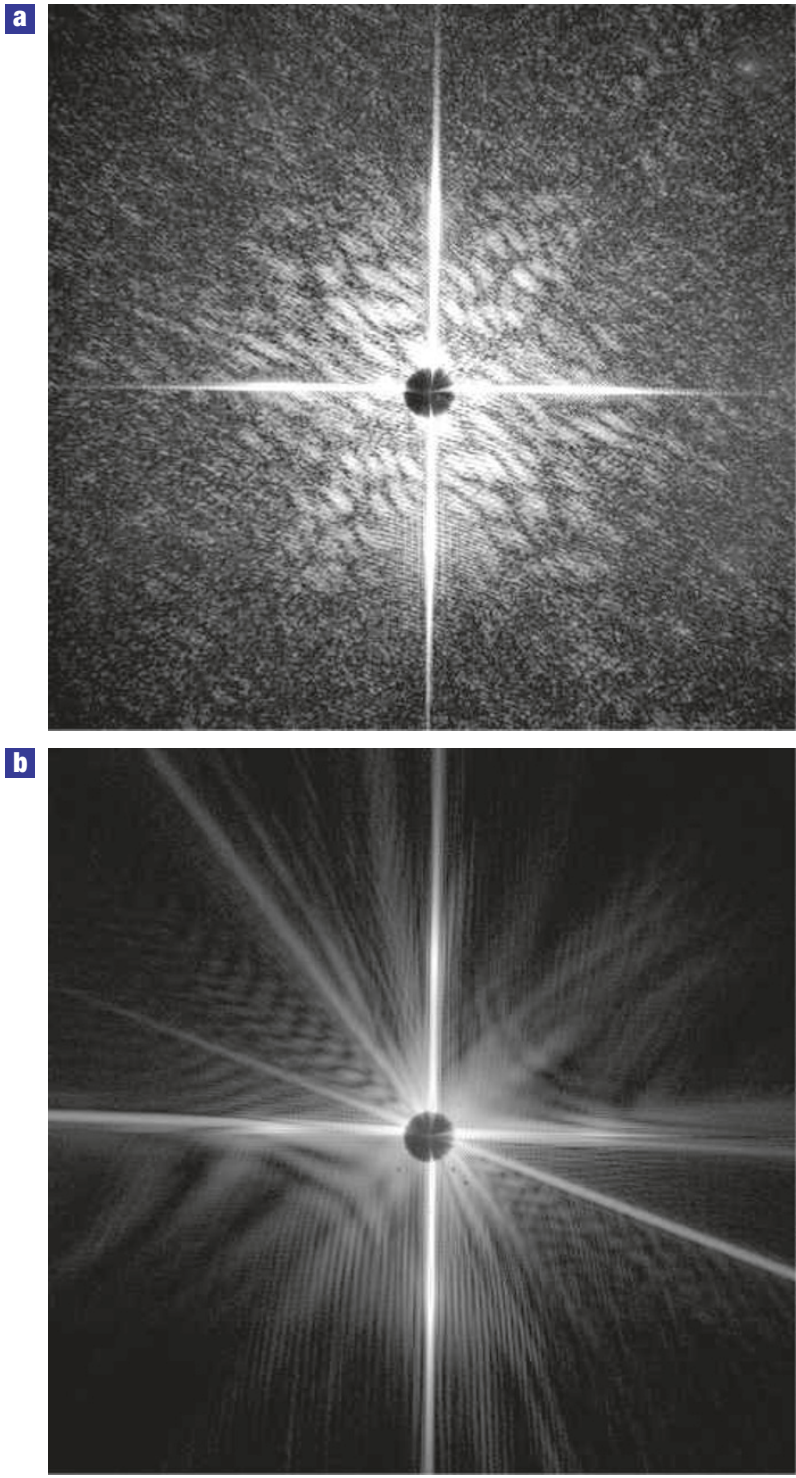

Figure 2 Flash X-ray coherent diffraction patterns. a,b, Coherent diffraction patterns recorded for a single $(4 \pm 2) \times 10^{14} \mathrm{~W} \mathrm{~cm}^{-2}, 25 \pm 5 \mathrm{fs}$ pulse (a) and for the subsequent pulse of similar intensity and duration, $20 \mathrm{~s}$ later (b), showing diffraction from the damage caused by the pulse that formed $\mathbf{a}$. The intensity is shown on a logarithmic greyscale, with black denoting one photon per pixel and white denoting 2,000 photons per pixel for $\mathbf{a}$ and 50,000 photons per pixel for $\mathbf{b}$. The entire patterns are shown as detected by the CCD, and extend to a diffraction angle of $15^{\circ}$ at the midpoint of the edges (corresponding to a momentum transfer of $8.1 \mu \mathrm{m}^{-1}$ ).

reference. Image reconstruction was carried out by phase retrieval using our iterative transform algorithm, Shrinkwrap ${ }^{8}$ (see the Methods section). Unlike similar algorithms ${ }^{7,20-23}$, Shrinkwrap solves the phase problem without requiring any a priori knowledge about the object.

The ultrafast coherent diffraction pattern of a nanostructured non-periodic object is shown in Fig. 2a. The object was a micrometre-sized pattern cut through a partially transparent silicon nitride membrane with a focused ion beam (FIB), and it is shown in the inset of Fig. 1. The pattern extends to a diffraction angle of $15^{\circ}$ at the midpoint of its edge. On the basis of low-fluence optical parameters ${ }^{24}$, we estimate ${ }^{3,25}$ that the absorbed energy 

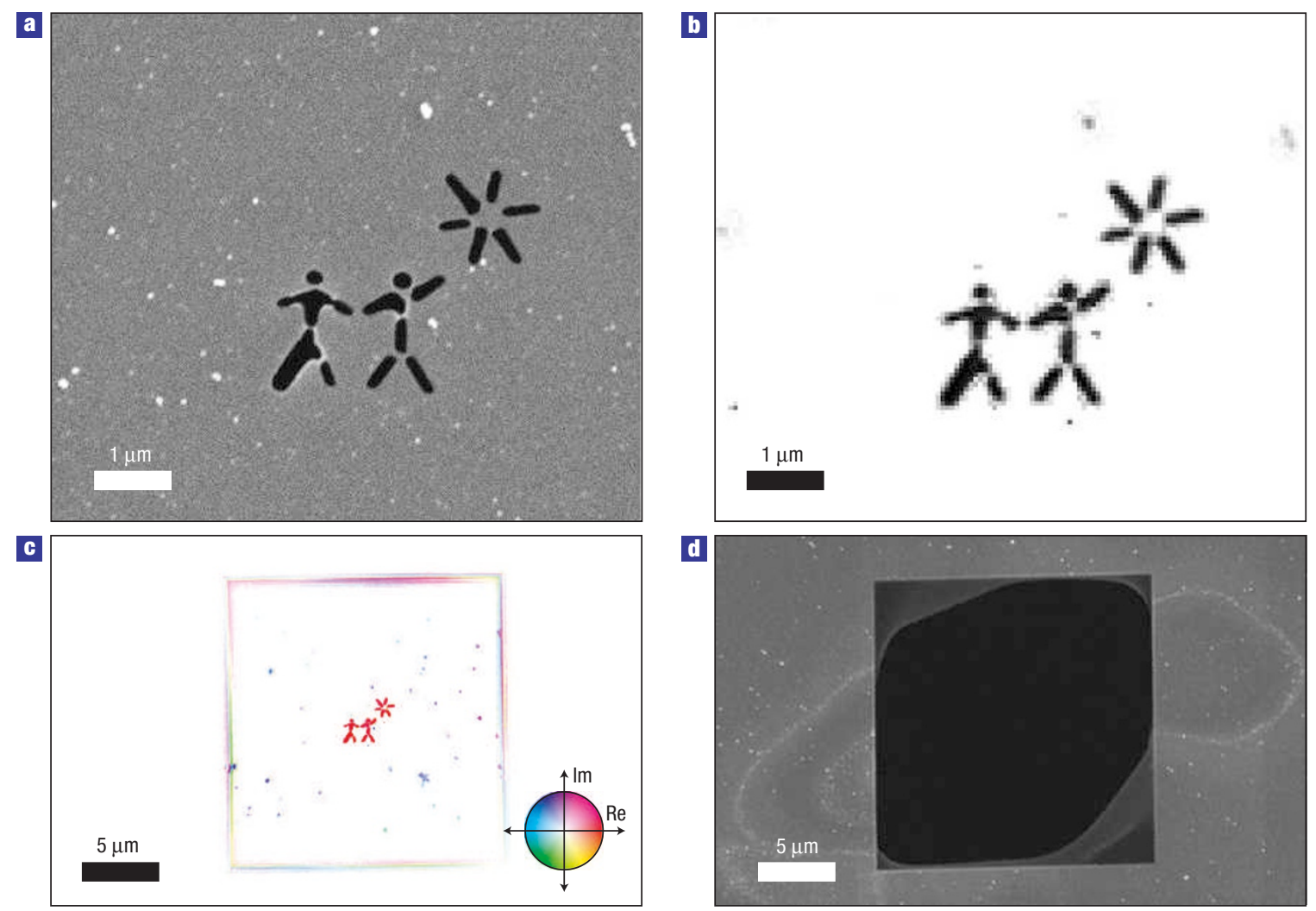

Figure 3 The reconstructed X-ray image shows no evidence of the damage caused by the pulse. a, SEM image of the sample before exposure to the FEL beam. The 20-nm-thick sample was held in a square supporting window that is $20 \mu \mathrm{m}$ wide. b,c, Image reconstructed, from the ultrafast coherent diffraction pattern of Fig. 2a, by phase retrieval and inversion using the Shrinkwrap algorithm ${ }^{8}$. In $\mathbf{b}$ the squared modulus of the retrieved complex image is shown on a linear greyscale from zero scattered photons per pixel (white) to $1.5 \times 10^{6}$ scattered photons per pixel (black), and $\mathbf{c}$ shows the complex image, with complex values on the Argand plane mapping to colour by saturation (absolute value) and hue (phase) according to the colour wheel shown. Pixel size in the reconstruction equals $62 \mathrm{~nm}$, corresponding to the half-period of the finest spatial frequency that can be recorded on our camera at $32 \mathrm{~nm}$ wavelength. The retrieved image clearly shows the silicon-window edge (in c), the FIB pattern and putative dirt particles, many of which correspond to the SEM image and which have an $80-100^{\circ}$ phase shift relative to the FIB pattern. $\mathbf{d}$, SEM image of the test sample after the exposures to the FEL beam, showing the square $20 \mu \mathrm{m}$ window and some remaining silicon nitride, as well as visible damage to the silicon support caused by the non-circular beam.

density was approximately $20 \mathrm{eV}$ per atom in the silicon nitride and that the sample reached a temperature of about $60,000 \mathrm{~K}$ before vaporizing. A second diffraction pattern taken $20 \mathrm{~s}$ after the first exposure is shown in Fig. 2b. This shows diffraction from a hole left in the membrane caused by the first pulse. That is, the first pulse utterly destroyed the sample, but not before a diffraction pattern of the apparently undamaged object could be recorded. Images of the object obtained with a scanning electron microscope (SEM), before and after FEL exposure, are shown in Fig. 3.

The main features of the diffraction pattern of Fig. 2a are speckles and strong vertical and horizontal lines that pass through the centre of the pattern. The horizontal and vertical lines are caused by interference of the waves diffracting from the opposite edges of the square window frame that holds the silicon nitride membrane. The speckles correspond to two length scales of the sample. The modulations of $\sim 60$ pixels (measured diagonally) in the diffraction pattern near the centre correspond to the narrow $2.5 \mu \mathrm{m}$ diagonal dimension of the object, and the finer speckles of about 16 pixels correspond to the distance between the picture object and the window frame in which it is centred. The speckles remain well defined out to the edge of the detector, although their visibility diminishes with scattering angle. This may be due to the fact that, at the high diffraction angles at the edge of the charge-coupled device (CCD) detector, the optical path difference between rays diffracting from points in the object transversely separated by $20 \mu \mathrm{m}$ (the sample window size) is $\sin 15^{\circ} \times 20 \mu \mathrm{m}=5 \mu \mathrm{m}$. This is comparable to the length of a $25 \mathrm{fs}$ pulse, which is $7.5 \mu \mathrm{m}$. That is, the overlap of the beams in time (and hence interference between them) occurs for only one-third of the pulse at high angles. The fact that we do observe interference over these optical path delays supports the notion ${ }^{17}$ that the FEL pulses are close to transform limited.

Figure $3 \mathrm{~b}, \mathrm{c}$ shows the image of the object reconstructed directly from the diffraction pattern of Fig. 2a. The modulus squared of the complex-valued image, which is proportional to the number of scattered photons within the detector acceptance, is shown in Fig. 3b. In Fig. 3c we show the complex-valued image, in a colour space where hue represents the phase and saturation the absolute value. The angular acceptance, $\alpha$, of our detector is $15^{\circ}$ at the midpoint of the detector edges, and $20^{\circ}$ at the corners. The diffraction-limited resolution length is $\lambda /(2 \sin \alpha)=62 \mathrm{~nm}$ for a wavelength of $\lambda=32 \mathrm{~nm}$. This length is defined as the half-period of the finest spatial frequency in the image, equal to an image pixel width. Along diagonal directions, the increased CCD acceptance gives a resolution length of $43 \mathrm{~nm}$. The actual image resolution would be worse than the diffraction limit if 


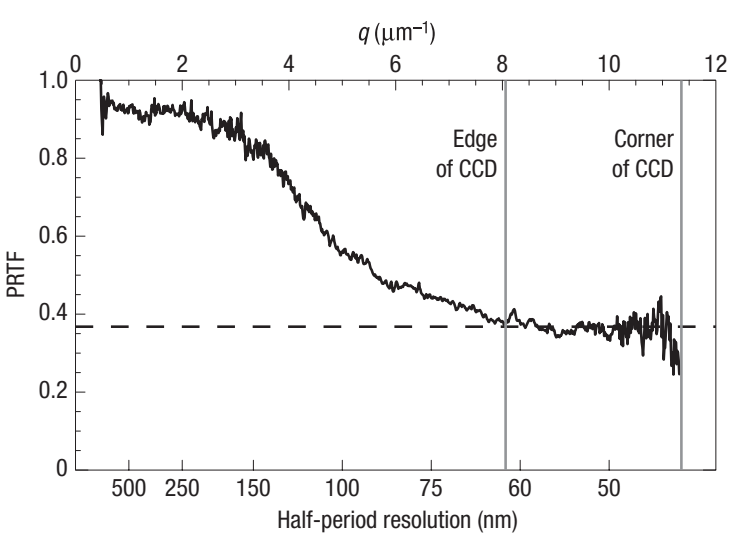

Figure 4 The image reconstructed to the diffraction limit. PRTF 9,23 for the reconstructed image shown in Fig. 3b,c, averaged over shells of constant momentum transfer $q=(2 / \lambda) \sin (\alpha / 2)$, where $\lambda$ is the wavelength and $\alpha$ the diffracted angle. The value of the PRTF is equal to unity where the phase is consistently retrieved, and zero where the phase is unknown. The resolution length of our image is $62 \mathrm{~nm}$, where the PRTF drops to a value of $1 / \mathrm{e}$.

the retrieved phases were incorrect, in the same way that phase errors in a lens cause image aberrations. We therefore estimate the image resolution by computing the phase-retrieval transfer function (PRTF $)^{9,23}$, shown in Fig. 4. This function represents the confidence with which the diffraction phases have been retrieved, and is calculated by comparing the Fourier amplitudes of the average of multiple independent reconstructions with the measured diffraction amplitudes. Where the phase of a particular Fourier component is consistently retrieved, the complex values add in phase, whereas if the phase is random the sum will approach zero. The PRTF is thus equal to unity when the phase is consistently retrieved and zero when the phase is unknown. We define the resolution of our reconstructed image according to where the PRTF drops to $1 / \mathrm{e}$ (ref. 23), or, if the PRTF does not drop below $1 / \mathrm{e}$ within the angular acceptance of the detector, we claim diffraction-limited resolution. For this image the resolution length is $62 \mathrm{~nm}$. We note that the same experimental geometry deployed on a hard-X-ray FEL operating at $0.15 \mathrm{~nm}$ wavelength would yield a diffraction-limited resolution length of $0.3 \mathrm{~nm}$.

The 'lensless' imaging method used here can be extended to atomic resolution, which will require shorter-wavelength X-rays and tighter focusing than demonstrated here. Hard-X-ray FELs are currently being developed that will create pulses that, when focused on the sample, will produce photon intensities five orders of magnitude higher than used here. An understanding of photonmaterial interactions on ultrashort timescales and at high X-ray intensities is fundamentally important to all experiments with $\mathrm{X}$-ray lasers. This area of science is virtually unexplored. The FLASH FEL in Hamburg is the first radiation source to permit experiments near the relevant photon energies and intensities. Our present results validate the concept of single-shot imaging with extremely intense and ultrashort soft-X-ray pulses, which are capable of destroying anything in their path. The resulting diffraction pattern carries high-resolution structural information about the object, and the resolution of the reconstructed image extends to the diffraction limit. This indicates that significant damage occurs only after the ultrashort $25 \mathrm{fs}$ FEL pulse traverses the sample, although more experiments will be necessary, when shorter-wavelength X-ray lasers become available, to determine how high a resolution flash imaging can be applied. These results have implications for studying non-periodic molecular structures in biology, or in any other area of science and technology where structural information with high spatial and temporal resolution is valuable. They also point to the viability of nanometre- to atomicresolution imaging of non-periodic and non-crystalline objects ${ }^{1-4}$ with hard-X-ray FELs.

\section{METHODS}

Samples consisted of a 20-nm-thick silicon nitride membrane spanning a $20-\mu \mathrm{m}$-wide square silicon window. We cut the pattern through the membrane with a dual-beam FIB instrument (FEI, National Center for Electron Microscopy, Lawrence Berkeley National Laboratory), using a 500 pA beam of $30 \mathrm{keV} \mathrm{Ga}^{+}$ions. The 20 -nm-thick silicon nitride membrane has a transmission of $44 \%$ at a wavelength of $32 \mathrm{~nm}$, and causes an estimated phase advance of $20^{\circ}$ relative to propagation through the same length of vacuum, as calculated from the known low-fluence optical constants ${ }^{24}$.

We carried out experiments in vacuo, as everything in the direct beam contributes to the diffraction pattern. We placed the samples in a vacuum vessel $70 \mathrm{~m}$ from the FEL undulator. We focused the FEL pulse to a $30 \mu \mathrm{m} \times 20 \mu \mathrm{m}$ focal spot on the sample with a 2-m-focal-length ellipsoidal mirror in the beam line. We selected single pulses with a fast shutter. Owing to the source coherence, we did not need an aperture to select a coherent patch of the beam, nor did we use a monochromator to select a narrow wavelength band of the radiation.

A novel X-ray camera was developed to record low-noise diffraction data from the sample in the forward direction (see Fig. 1). In this camera, a graded multilayer plane mirror separates the diffracted beam from the direct beam, and the intense direct beam passes harmlessly through the hole in the centre of the mirror without damaging the detector. The diffracted light reflects onto a back-illuminated direct-detection CCD chip (Princeton Instruments, in-vacuum PI-MTE CCD), containing 1,300 $\times 1,340$ square pixels of $20 \mu \mathrm{m}$ width. The resonant X-ray multilayer of the planar mirror consists of layers of $\mathrm{Si}, \mathrm{Mo}$ and $\mathrm{B}_{4} \mathrm{C}$, and was fabricated so that the layer period varies from 18 to $32 \mathrm{~nm}$ across the mirror. The variation in multilayer period matches the variation in the angle of incidence of rays emanating from the sample that strike the mirror. This angle varies from 30 to $60^{\circ}$, as depicted by the contour lines on the mirror in Fig. 1. We achieved the gradient by sputter-depositing the multilayer materials through a mask onto the rotating substrate, so that the time-averaged deposition gave the desired material thickness at each point on the mirror. The $32 \mathrm{~nm}$ reflectivity across the mirror was $45 \%$, as measured at a synchrotron-based reflectometer ${ }^{26}$. The bandwidth of the $32 \mathrm{~nm}$ pulse was $0.2 \mathrm{~nm}$, and the reflectance bandwidth of the multilayer mirror was $9 \mathrm{~nm}$, centred at $32 \mathrm{~nm}$. The sample is heated up to a plasma of about $7 \mathrm{eV}(60,000 \mathrm{~K})$, following exposure, and will emit significant blackbody radiation extending beyond $50 \mathrm{eV}$. The resonant mirror filters out most of this radiation. Also, off-axis radiation scattered from beamline components is reflected at less than $1 \%$ and hence filtered from the diffraction pattern. The reflectivity of the coating diminishes smoothly to zero close to the edge of the central hole, owing to decoherence of the coating layers caused by the underlying substrate roughness where the hole has been cored. This 'soft edge' reduces scatter from the hole, whose shadow we can see as a dark circle at the centre of the patterns in Fig. 2. The on-axis path length of the reflected beam from the sample to the CCD was $55 \mathrm{~mm}$, and for $32 \mathrm{~nm}$ radiation and objects smaller than $20 \mu \mathrm{m}$ this distance is in the far field, where the diffraction pattern is equal to the Fourier transform of the exit wave ${ }^{27}$.

Image reconstruction was carried out with the Shrinkwrap algorithm ${ }^{8}$. Phase retrieval in Shrinkwrap is a nonlinear optimization problem in a high-dimensional phase space. The dimensionality is equal to the number of phases to be retrieved: 1.7 million in this case. We obtain the solution iteratively by sequentially enforcing known constraints in diffraction and image spaces. We specifically aim for diffraction phases that are such that the waves re-interfering to form the image must all destructively cancel in areas outside the object's boundary (called its support), and that the amplitudes of the discrete Fourier transform of the image match the measured diffraction amplitudes (which must be measured finely enough to include enough empty space beyond the object to constrain the phases). Other iterative transform algorithms usually require that the support of the object be known a priori, and the closer the support to the actual object boundary, the better the reconstruction. Shrinkwrap, however, periodically refines the support 
constraint from the current estimate of the image. The support constraint is calculated every 70 iterations by selecting pixels with intensity values greater than 0.2 times the maximum image intensity, after first blurring the image with a gaussian kernel. We initially set the blurring kernel to 3 pixels full-width at half-maximum, and gradually reduce it to 0.7 pixels full-width at half-maximum by iteration 5,000. The final support is that found four update cycles before the point where the normalized image error ${ }^{9}$ exceeds a value of 0.2 . This stopping criterion is typically reached in 3,000-4,000 iterations. During the iterations we did not constrain the intensity or phase in the region in the mirror hole, which contains the unrecorded zero spatial frequency, nor did we constrain the object to be real or positive.

Once the diffraction phases are retrieved, the inverse Fourier transform of the complex-valued diffraction pattern yields the complex-valued image of the wavefield exiting the sample. The modulus squared of the wavefield, equal to the number of photons per pixel that are scattered into the solid angle of acceptance of the detector, is shown in Fig. 3b. The lost information in the hole includes the lowest spatial frequencies (components of periods $2.0 \mu \mathrm{m}$ and longer), which are thus missing from this image. In microscopy, such images are known as dark-field images, as the featureless background of the object does not contribute to the angularly scattered radiation and therefore is dark in the image. Additionally, the complex-valued image is known only to a constant arbitrary phase, and phase differences can be measured only where there is scattered intensity. In the colour-encoded complex image in Fig. 3c, we have set the arbitrary phase offset so that the phase through the FIB structures is zero.

We carried out many reconstructions, starting each time from random phases. Each reconstructed image varied slightly, owing to the fact that with photon shot noise there is no true solution that exactly satisfies all constraint sets. However, each image determined from the final iterate was clearly recognizable as compared with the SEM image. The image estimate shown in Fig. 3 b,c is the average of 250 independent reconstructions.

\section{Received 15 September 2006; accepted 16 October 2006; published 12} November 2006.

\section{References}

1. Neutze, R., Wouts, R., van der Spoel, D., Weckert, E. \& Hajdu, J. Potential for biomolecular imagin with femtosecond X-ray pulses. Nature 406, 752-757 (2000).

2. Jurek, Z., Faigel, G. \& Tegze, M. Dynamics in a cluster under the influence of intense femtosecond hard x-ray pulses. Eur. Phys. J. D 29, 217-229 (2004).

3. Hau-Riege, S. P., London, R. A. \& Szöke, A. Dynamics of X-ray irradiated biological molecules. Phys. Rev. E 69, 051906 (2004)

4. Bergh, M., Timneanu, N. O. \& van der Spoel, D. Model for the dynamics of a water cluster in an X-ray free electron laser beam. Phys. Rev. E 70, 051904 (2004).

5. Fienup, J. R. Phase retrieval algorithms—a comparison. Appl. Opt. 21, 2758-2769 (1982).

6. Sayre, D., Chapman, H. N. \& Miao, J. On the extendibility of $\mathrm{x}$-ray crystallography to noncrystals. Acta Crystallogr. A 54, 232-239 (1998).

7. Miao, J., Charalambous, P., Kirz, J. \& Sayre, D. Extending the methodology of $\mathrm{x}$-ray crystallography to allow imaging of micrometre-sized non-crystalline specimens. Nature 400, 342-344 (1999).

8. Marchesini, S. et al. X-ray image reconstruction from a diffraction pattern alone. Phys. Rev. B 68 140101 (2003).

9. Chapman, H. N. et al. High-resolution ab initio three-dimensional X-ray diffraction microscopy J. Opt. Soc. Am. A 23, 1179-1200 (2006).

10. Huldt, G., Szöke, A. \& Hajdu, J. Diffraction imaging of single particles and biomolecules. J. Struct. Biol. 144, 219-227 (2003)

11. Henderson, R. The potential and limitations of neutrons, electrons and X-rays for atomic resolution microscopy of unstained biological molecules. Quart. Rev. Biophys. 28, 171-193 (1995).
12. Howells, M. R. et al. An assessment of the resolution limitation due to radiation-damage in $\mathrm{x}$-ray diffraction microscopy. J. Electron. Spectrosc. Relat. Phenom. (in the press); Preprint at $<$ http://arxiv.org/abs/physics/0502059> (2005).

13. Persson, P., Lunell, S., Szoke, A., Ziaja, B. \& Hajdu, J. Shake-up and shake-off excitations with associated electron losses in X-ray studies of proteins. Protein Sci. 10, 2480-2484 (2001).

14. Timneanu, N., Caleman, C., Hajdu, J. \& van der Spoel, D. Auger electron cascades in water and ice. Chem. Phys. 29, 277-283 (2004)

15. Ziaja, B., London, R. A. \& Hajdu, J. Unified model of secondary electron cascades in diamond J. Appl. Phys. 97, 064905 (2005)

16. Solem, J. C. \& Baldwin, G. C. Microholography of living organisms. Science 218, 229-235 (1982).

17. Ayvazyan, V. et al. First operation of a free-electron laser generating GW power radiation at $32 \mathrm{~nm}$ wavelength. Eur. Phys. J. D 37, 297-303 (2006).

18. Saldin, E. L., Schneidmiller, E. A. \& Yurkov, M. The Physics of Free-Electron Lasers (Springer, Berlin, 2000).

19. Wolf, E. Three-dimensional structure determination of semi-transparent objects from holographic data. Opt. Commun. 1, 153-156 (1969).

20. Pfeifer, M. A., Williams, G. J., Vartanyants, I. A., Harder, R. \& Robinson, I. K. Three-dimensional mapping of a deformation field inside a nanocrystal. Nature 442, 63-66 (2006).

21. He, H. et al. Experimental lensless soft-x-ray imaging using iterative algorithms: Phasing diffuse scattering. Acta Crystallogr. A 59, 143-152 (2003).

22. Miao, J. et al. Imaging whole Escherichia coli bacteria by using single particle $\mathrm{x}$-ray diffraction. Proc. Natl Acad. Sci. USA 100, 110-112 (2003).

23. Shapiro, D. et al. Biological imaging by soft x-ray diffraction microscopy. Proc. Natl Acad. Sci. USA 102, 15343-15346 (2005).

24. Henke, B. L., Gullikson, E. M. \& Davis, J. C. X-ray interactions: photoabsorption, scattering, transmission, and reflection at $E=50-30000 \mathrm{eV}, Z=1-92$. At. Data. Nucl. Data. Tables 54, 181-342 (1993).

25. More, R. M., Warren, K. H., Young, D. A. \& Zimmerman, G. B. A new quotidian equation of state (QEOS) for hot dense matter. Phys. Fluids 31, 3059-3078 (1988).

26. Underwood, J. H. \& Gullikson, E. M. High-resolution, high-flux, user friendly VLS beamline at the ALS for the 50-1300 eV energy region. J. Electron. Spectrosc. Relat. Phenom. 92, 265-272 (1998).

27. Goodman, J. W. Statistical Optics (Wiley, New York, 1985).

\section{Acknowledgements}

We owe special thanks to the scientific and technical staff of FLASH at The Deutsches Elektronen-Synchrotron, Hamburg, in particular to J. Feldhaus, R. L. Johnson, U. Hahn, T. Nuñez, K. Tiedtke, S. Toleikis, E. L. Saldin, E. A. Schneidmiller and M. V. Yurkov. We also thank R. Falcone, M. Ahmed and T. Allison for discussions, J. Alameda, E. Gullikson, F. Dollar, T. McCarville, F. Weber, J. Crawford, C. Stockton, W. Moberlychan, M. Haro, A. Minor, H. Thomas and E. Eremina for technical help with these experiments. The following agencies supported this work: the US Department of Energy (DOE) under contract to the University of California, Lawrence Livermore National Laboratory (the project was funded by the Laboratory Directed Research and Development Program at LLNL); The National Science Foundation Center for Biophotonics, University of California, Davis; The National Center for Electron Microscopy and the Advanced Light Source, Lawrence Berkeley Laboratory; Natural Sciences and Engineering Research Council of Canada (NSERC Postdoctoral Fellowship to M.J.B.); Sven and Lilly Lawskis Foundation (doctoral fellowship to M.M.S.); the US Department of Energy Office of Science to the Stanford Linear Accelerator Center; the European Union (TUIXS); The Swedish Research Council; The Swedish Foundation for International Cooperation in Research and Higher Education and The Swedish Foundation for Strategic Research. Correspondence and requests for materials should be addressed to H.N.C. or J.H.

\section{Author contributions}

H.N.C. and J.H. conceived the experiment and H.N.C., A.B., M.J.B., M.F., S.P.H.-R., S.M., B.W.W., S. Bajt, W.H.B., R.A.L., R.W.L., A.S., K.O.H., C.B., T.M. and J.H. contributed to its design. S. Bajt, E.S. and H.N.C. designed the multilayer optics. S.B. designed and fabricated the samples and S.B. and M.J.B. characterized them. E.P., M.K., R.T., S.D., T.T. and J.R.S. carried out interfacing and optimization of the experiment with FLASH. H.N.C., A.B., M.J.B., S.B., M.F., S.M., B.W.W., W.H.B., E.P., M.K., R.T., S.D., T.T., T.M., C.B., M.H., D.A.S., F.B., M.B., C.C., G.H., M.M.S. and J.H. carried out the experiment and H.N.C., A.B., M.J.B., S.B., S.P.H.-R., S.M., D.v.d.S., F.B., M.B., C.C., G.H., M.M.S., F.R.N.C.M., A.S., N.T. and J.H. carried out data analysis and interpretation. All authors discussed the results and contributed to the final manuscript.

Competing financial interests

The authors declare that they have no competing financial interests.

Reprints and permission information is available online at http://npg.nature.com/reprintsandpermissions/ 